\section{Case Reports in Ophthalmology}

\title{
Bilateral Giant Retinal Tear and Sequential Vitrectomy
}

\author{
Mushawiahti Mustapha $^{a} \quad$ Edward Roufail Franzco $^{b}$ \\ aDepartment of Ophthalmology, Universiti Kebangsaan Malaysia Medical Centre,

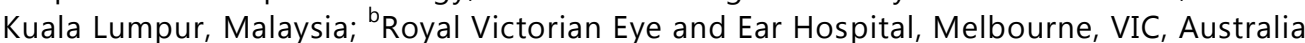

\section{Keywords}

Endotamponades · Perfluorocarbon liquid · Retinal detachment · Retinal tear · Silicone oil · Vitrectomy

\begin{abstract}
Purpose: To describe the excellent outcome of surgery for bilateral giant retinal tears (GRTs) with better options of endotamponade. Methods: This is a case report of a 62-year-old man who presented with bilateral GRTs and associated retinal detachment. The tear in the right eye was supero-temporal and silicone oil was used as an endotamponade. The tear in the left eye was infero-temporal and perfluorocarbon liquid was used as an endotamponade. $\boldsymbol{R e}$ sults: The outcome at 6 months after surgery was excellent with visual acuities of $6 / 6$ in both eyes. Conclusion: Improved availability of endotamponade agents allows repair of bilateral GRTs to be done at the same time, with good surgical outcomes.

(C) 2017 The Author(s)

Published by S. Karger AG, Basel
\end{abstract}

\section{Introduction}

Giant retinal tear (GRT) is a full thickness retinal tear that extends for more than $90^{\circ}$ circumferentially [1]. It can rapidly lead to extensive retinal detachment with potentially severe proliferative vitreoretinopathy (PVR). This results in severe scarring, predisposing to surgical failure. It often occurs in "at risk" eyes, with a particular association with myopia [2, 3]. In 


\section{Case Reports in Ophthalmology}

$3.2 \%$ of cases, GRTs are bilateral at presentation [2]. The mean age at presentation is 39.6 years, but it has been reported to occur in those as young as 8 years of age [2].

GRT poses a considerable management challenge to the vitreoretinal surgeon [1]. Both the tear itself and the associated detached retina contribute to the high risk of PVR and the failure of retinal reattachment [2]. A number of surgical techniques have been proposed to treat GRT, including isolated vitrectomy and the combined buckle procedure. Some advocate removal of the lens, even when it is clear [2]. Intraoperative challenges can arise as a consequence of the size of the tear, a large retinal fold, and slippage [2-4]. There is a high failure rate with primary vitrectomy.

Following primary surgery, a successful reattachment rate of $71 \%$ has been reported and the prognosis for vision is poor [2,3]. In one study, vision was found to have improved following surgery in only $37.6 \%$ of patients and in only $52 \%$ of patients was vision better than 20/200 [5]. Besides the involvement of the macula, the main determinants of the final visual acuity are the status of the lens, aphakia, and alteration in the axial length of the globe caused by placement of a buckle $[2,5,6]$.

Silicone oil or long-acting gas have been the preferred tamponading agents when treating GRT $[2,6]$. Perfluorocarbon liquid was developed to facilitate vitreoretinal surgery intraoperatively.It has been said that it has become the surgeon's third hand for complex cases [5]. Its particular properties have also led to its use as a short-term endotamponade [3].

We report a case of bilateral GRT with associated retinal detachment undergoing surgery for both eyes at the same setting with good surgical and visual outcomes. The availability of more and better options for endotamponade allowed a successful outcome in this case. The study was conducted at the Royal Victorian Eye and Ear Hospital, Melbourne, VIC, Australia.

\section{Case Report}

A 62-year-old man presented with sudden loss of vision in his right eye of a day's duration. Vision was reduced to counting fingers in the right eye and was $6 / 6$ in the left eye. There was no family history of retinal detachment, he was not myopic, and he had no features to suggest a syndromic diagnosis. The anterior segments and lenses in both eyes were normal. Examination of the right fundus revealed a $100^{\circ}$ supero-temporal GRT with associated retinal detachment involving the macula. The tear was associated with a long circumferential lattice with no evidence of PVR. The retina was fairly mobile and the detachment appeared very recent. Examination of the left fundus revealed a $120^{\circ}$ infero-temporal GRT with associated retinal detachment sparing the macula. Again, there was no evidence of PVR. He had not reported any symptoms in the left eye.

Surgery on both eyes was performed under general anesthesia on the same day. Bilateral vitrectomy was performed using the 23-gauge system with peripheral trimming of the vitreous gel. The retinae were unfolded and stabilized using perfluorocarbon liquid (Perfluron; Alcon, Fort Worth, TX, USA) followed by $360^{\circ}$ endolaser retinopexy. Given the superotemporal location of the tear, the right eye procedure was completed using a direct perfluorocarbon liquid-silicone oil exchange. Silicone oil was used as an endotamponade at the end of the surgery. The procedure to the left eye, given the infero-temporal location of the tear, was completed using perfluorocarbon liquid left in situ. Following surgery, the patient was encouraged to stay in an upright position. 


\section{Case Reports in Ophthalmology}

Two weeks later, the perfluorocarbon liquid was removed from the left eye and the eye was filled with gas (SF6 30\%). At the time of operation, the retina appeared stable with no features of PVR. At 3 months the silicone oil was removed from the right eye, combined with cataract extraction and intraocular lens implantation. At 6 months after surgery, the retinae in both eyes remained stable and the visual acuities were 6/6.

\section{Discussion}

Surgical advances and the availability of new endotamponade agents hold the promise of improved outcome following bilateral concurrent surgical treatment of GRT. Silicone oil is considered superior to short-acting gas as an endotamponade in such cases $[2,5,6]$. Complete filling with silicone oil is important in order to achieve a good tamponade. Traditionally, large inferior tears were treated with silicone oil tamponade. However, as a result of the low molecular weight of silicone oil compared to water, it tends to float upwards, impairing the tamponade effect with inferior tears. Even when complete filling is achieved, with time the emulsification of the oil into small droplets creates a space inferior to the oil globule. As a consequence, the development of inferior PVR caused by an accumulation of inflammatory mediators underneath the floating oil is unavoidable. This proliferation of scar tissue causes retinal detachment, thus causing the patient to be at a high risk of surgical failure [7].

Perfluorocarbon liquid is a new-generation vitreous substitute. It is heavier than water and silicone oil. It has high specific gravity, low viscosity, immiscibility, optical clarity, high surface tension with water, and it is inert [5]. Originally, it was used to help stabilize and assist the unfolding of the retina during surgery [5]. There has been concern about the potential of perfluorocarbon liquid used as an endotamponade to cause histological changes of retinal pigment epithelium due to its heavy mechanical profile [8]. However, recently its successful and safe use as a temporary endotamponade has been reported $[3,9]$. It is generally recommended that it is used as an endotamponade for no longer than 2 weeks and then requires surgical removal [9]. As a result of its high specific gravity,it can be expected to have a superior tamponading effect in comparison to silicone oil, when the pathology is inferior within the globe. This may lead to an improved surgical outcome with high-risk inferior GRTs. Visual outcome was reported to be excellent in a perfluorocarbon liquid tamponade case series reported by Drury and Bourke [9]. Visual acuity improved in up to $86 \%$ of cases which involved macular retinal detachment associated with GRT. In their series there was an absence of macular damage.

Our case demonstrated the safe and successful use of different endotamponades for bilateral sequential vitrectomy due to GRT. Preoperatively, the patient's potential for visual recovery was considered good because the retinae were mobile, the detachments appeared fresh, and there was no evidence of PVR. Based on the position of the GRTs, we used silicone oil as an endotamponade for the right eye to treat a superior tear and perfluorocarbon liquid for the left eye to treat an inferior tear. Upright posture was encouraged to assist the tamponade effect in both eyes and was well tolerated by the patient. This not only helps with the tamponading effect, but is also more comfortable for the patient as the face-down position or maintenance of a head tilt is unnecessary.

Bilateral GRT is an uncommon condition, and achieving a good anatomical and visual outcome with simultaneous surgery to both eyes represents a considerable challenge. The unique aspect of this case was the different locations of the GRTs in the 2 eyes. The differing properties of the tamponading agents used was key to successful surgical treatment. 
The availability of various types of endoptamponade with different properties means that we now have a range of treatment options, allowing the surgeon to individualize his approach. In particular, it is possible to perform concurrent surgery in both eyes with the expectation, in selected patients, of excellent anatomical and functional outcomes.

\section{Statement of Ethics} journal.

The patient/next of kin/guardian consented to the submission of the case report to the

\section{Disclosure Statement}

All authors certify that they have no affiliations with or involvement in any organization or entity with any financial interest (such as honoraria, educational grants, or other equity interest) or nonfinancial interest (such as personal or professional relationships, affiliations, knowledge, or beliefs) in the subject matter or materials discussed in this manuscript.

\section{References}

$\checkmark 1$ Schepens CL, Dobbie JG, McMeel JW: Retinal detachments with giant retinal breaks: preliminary report. Trans Am Acad Ophthalmol Otolaryngol 1962;66:471-478.

2 Lee SY, Ong SG, Wong DWK, Ang CL: Giant retinal tear management: an Asian experience. Eye 2009;23:601-605.

3 Roufail ML, Lawrence R: Perfluoro-n-octane as a postoperative vitreoretinal tamponade in the management of giantretinal tears. Retina 2005;25:897-9016.

-4 Ruiz CQ; Hidalgo RA, Cirujanos CY: Giant retinal tears treated with lens sparing, bimanual $23 \mathrm{~g}$ vitrectomy without scleral buckle (in Spanish). Cir Cir 2014;82:245-251.

-5 Al-Khairi AM, Al-Khatani E, kangave D, et al: Prognostic factors associated with outcomes after giant retinal tears management using perfluorocarbon liquids. Eur J Ophthalmol 2008;18:270-274.

-6 Dabour SA: The outcome of surgical management for giant retinal tear more than $180^{\circ}$. BMC Ophthalmol 2014;27:86.

7 Asaria RHY, Kon CH, Bunce C, et al: Silicone oil concentrates fibrogenic growth factors in the retro-oil fluid. Br J Ophthalmol 2004;88:1439-1442.

-8 Stolba U, Krepler K, Velikay-Parel M, et al: The effect of specific gravity of perfluorocarbon liquid on the retina after experimental vitreous substitution. Graefes Arch Clin Exp Ophthalmol 2004;242:931e6.

-9 Drury B, Bourke RD: Short-term intraocular tamponade with perfluorocarbon liquid. Br J Ophthalmol 2011;95:694-698. 